\title{
Tous ensemble contre la COVID-19
}

C hacun d'entre nous se souviendra du 16 mars 2020, de ce qu'il a fait de cette journée pour se préparer au mieux à la période qui allait suivre et dont nous ne soupçonnions ni la durée, ni le contenu, ni les difficultés engendrées.

Ainsi, à dater de ce jour, la quasi-totalité des collaborateurs de l'AFM-Téléthon est passée en télétravail complet. Une exception majeure toutefois pour les équipes du pôle Yolaine de Kepper, un ensemble de structures et services médico-sociaux situé à Angers et à sa périphérie. Il a fallu en effet poursuivre l'activité de la Maison d'Accueil Spécialisé (MAS) et de l'Habitat service Gâte-Argent tout en multipliant les précautions pour ne pas laisser la COVID-19 pénétrer auprès des résidents et des locataires de ces structures. Depuis le début mars et jusqu'à ce jour, la résidence Yolaine de Kepper est ainsi devenue une forteresse imprenable. Grâce à des mesures drastiques d'isolement et de prévention, la population des malades accueillis a, jusqu'à ce jour, échappé à la contamination. Nous avons vu des photos qui démontrent que la vie avec ses petits plaisirs a pu continuer malgré les contraintes du moment.

Pour les autres collaborateurs de l'AFM-Téléthon, soient environ 440 personnes, nous entamons, à l'heure de l'écriture de ces lignes, le $48^{e}$ jour de confinement à domicile et donc de télétravail.

L'adaptation s'est faite en 48 heures. Certes, le télétravail était déjà une pratique en place depuis quelques mois à l'AFM-Téléthon mais de façon périodique. Pour autant, il a fallu un engagement sans faille de la direction des services informatiques (DSI) pour équiper en matériel portable ceux qui ne l'étaient pas, déployer des logiciels de travail à distance, et accompagner ce virage numérique accéléré.

Les équipes de la mission Aider (actions familles, actions médicales, affaires publiques), et celles de la direction de la communication ont été, et sont toujours, en première ligne pour apporter aux malades et aux familles tous les conseils, recommandations, fiches techniques et tutoriels nécessaires pour bien se protéger et vivre au mieux le confinement. Avant même que le confinement ne devienne une obligation gouvernementale, la première de nos recommandations aux familles a été de rester au domicile, et si possible de limiter toute interaction avec l'extérieur en réduisant, voire en supprimant, le recours à des auxiliaires de vie professionnel(le)s ainsi qu'aux intervenants médicaux ou médico-sociaux. Ce n'était pas toujours possible du fait du degré de dépendance de la personne malade et des capacités d'action, parfois limitées, de son entourage familial ; néanmoins, la grande majorité l'a fait, avec pour retombée positive un très faible nombre de malades neuromusculaires touchés par la COVID-19, mais aussi, comme effet collatéral, une pression accrue sur les aidants familiaux.

Il ne fallait pas laisser pour autant les familles dans des situations d'isolement social. Le lien a pu être maintenu en mobilisant les ressources liées aux technologies de la communication et surtout un très fort engagement des réseaux professionnels et bénévoles de l'association: décryptage et traduction, à l'adresse des malades et des familles, des mesures gouvernementales par l'équipe des affaires publiques, rédaction de fiches pratiques et de fiches-conseils, élaboration de tutoriels, par exemple pour maintenir à domicile un peu d'entretien orthopédique. Le lien social a pu ainsi être maintenu: par téléphone (17 000 appels), mais aussi au travers des courriels, et des messages sur les réseaux sociaux, les sites internet et les blogs des groupes d'intérêt et des délégations... Les services régionaux de l'association, qui avaient suspendu très tôt les visites à domicile, ont poursuivi leur mission d'accompagnement des malades et des familles à distance, via le téléphone et les solutions de visio-conférence. C'est ainsi que de nouvelles familles ont pu faire l'objet d'une première visite "à domicile" en visio-conférence avec un retour d'expérience très positif.

Au total, sur le terrain, ce sont près de 120 Référents Parcours de Santé (RPS) déployés sur toute la France à partir de 18 Services régionaux, ainsi que les équipes bénévoles des délégations et groupes d'intérêt, qui sont mobilisés chaque jour pour apporter leur aide et leur soutien aux familles concernées.

Une cellule d'aide psychologique a pu être mise en place pour faire face à l'angoisse de certains malades ou de leur entourage familial et a mobilisé une dizaine de psychologues de l'Institut de Myologie, de l'hôpital Raymond Poincaré de Garches, du pôle Yolaine de Kepper et de l'AFM-Téléthon. Un dispositif venu en complément de la ligne Accueil Familles, elle-même déjà en place avant la pandémie et dont les effectifs ont été doublés en cette période. Cette assistance est joignable à tout moment de la journée, $7 \mathrm{~J} / 7$, pour répondre à tous les appels des malades et faire le lien avec l'astreinte médicale et psychologique. 
Cette période a permis de renforcer les liens et la collaboration entre le réseau associatif et les cliniciens au sein de la filière FILNEMUS

Les cliniciens et personnels des centres de référence et de compétences ont souvent été mobilisés pour renforcer les équipes dans les unités hospitalières dédiées à la COVID-19. Il a fallu revoir l'organisation hospitalière en isolant les unités neuromusculaires des unités dédiées à la COVID-19, et réorganiser les pratiques habituelles de prise en charge des malades neuromusculaires en soins courants. Les rendez-vous de consultations ou en hôpital de jour ont le plus souvent été reportés pour limiter les risques de contamination ; les examens et interventions non urgentes ont été différés. Il a fallu faire un tri des soins, examens et interventions en identifiant parmi eux, ceux qui ne pouvaient être différés sans mettre en danger le patient neuromusculaire. En parallèle, des solutions de téléconsultations ont pu être mises en place avec les patients et les familles dans beaucoup de centres de références ou de compétence qui ont pu ainsi maintenir le lien médical indispensable et atténuer l'impact de la crise épidémique, notamment dans les situations les plus à risque.

La concertation entre les cliniciens et les directions de l'AFM-Téléthon (actions médicales, actions auprès des familles, affaires publiques) a été exemplaire, constante, quasi quotidienne, l'idée étant de partager toutes les informations pour que les réseaux associatifs puissent s'en faire le porte-parole auprès des familles mais aussi pour leur apporter les réponses aux très nombreuses questions d'ordre médical posées dans le cadre de la prise en charge de leur maladie au vu du contexte épidémique.. Cette concertation a permis d'harmoniser les positions en adaptant une vision strictement médicale aux interrogations, avis et attentes des malades dont l'association est le porteparole.

Il s'est agi également de partager toutes les informations concernant les cas de patients neuromusculaires touchés par la COVID-19 (heureusement peu nombreux) et l'évolution de leur prise en charge et de leur maladie. Le recensement et la caractérisation de ces cas ont été réalisés dans le cadre de plusieurs enquêtes diligentées au niveau national (à l'initiative de FILNEMUS) et européen (grâce au réseau de référence Euro-NMD).

Une problématique cruciale a été la question de l'admission dans les unités de réanimation des malades neuromusculaires touchés par la COVID-19. Avoir une maladie neuromusculaire n'expose pas la personne à un risque majoré de contamination, avec un bémol toutefois pour les malades sous traitement immunosuppresseur. Les conséquences de l'infection pour les personnes à risque (notamment dans le cas d'une atteinte cardio-respiratoire pré-existante) restent toutefois à déterminer et sont fonction de la pathologie sous-jacente, de l'âge et du stade évolutif de la maladie. L'AFM-Téléthon en lien avec FILNEMUS a rédigé et diffusé une fiche technique pour les Centres 15: "Recommandations médicales COVID-19 et maladies neuromusculaires". Dans celle-ci, nous avons affirmé que toute personne atteinte d'une maladie neuromusculaire était éligible à une prise en charge par le SAMU-Centre 15, et en soins de réanimation en unité hospitalière si nécessaire. Avoir une maladie neuromusculaire ne saurait constituer un motif a priori de refus de soins, a fortiori dans le contexte de la COVID-19. FILNEMUS a publié dans la Revue Neurologique un article complet sur l'adaptation des pratiques médicales dans le contexte de l'épidémie [1]. La question de l'admission des patients neuromusculaires en réanimation y est abordée en listant les facteurs de bon pronostic en soins de réanimation en fonction des pathologies et des situations. Ce même article a permis de faire le point concernant les modalités d'administration et le maintien éventuel de traitements propres aux malades neuromusculaires, y compris les thérapies innovantes, ainsi que la conduite à tenir concernant les essais cliniques en cours. Afin d'aider concrètement les familles, l'AFM-Téléthon a mené de nombreuses actions pour doter de masques les familles et leurs aidants. L'accès aux masques et leurs modalités de distribution par les pouvoirs publics ont beaucoup évolue depuis le début de la crise sanitaire. L'AFM a mené une action forte de lobbying pour favoriser l'accès aux masques pour les malades et leurs aidants. Aujourd'hui, cet accès est possible pour les Services d'Accompagnement à Domicile via les Groupements Hospitaliers Territoriaux, et pour les aidants salariés en emploi direct via les pharmacies, même si cela ne fonctionne pas partout. Pour les malades et les aidants familiaux, l'AFM-Téléthon a donc effectué des demandes de masques région par région, auprès des Agences Régionales de Santé et parfois des Conseils Régionaux. À ce jour, les 14 régions ont répondu favorablement avec des stocks allant de 10000 à 100000 masques.

Depuis la mi-avril, l'AFM-Téléthon distribue les masques obtenus via ses Services régionaux avec l'appui des réseaux bénévoles, ses partenaires/prestataires intervenant auprès des familles, mais aussi le soutien des équipes de la CroixRouge Française Ile-de-France, sans oublier de nombreux partenaires logistiques avec un seul objectif : les remettre aux familles à risque qui en seraient dépourvues. Ces premiers lots de masques restent malheureusement insuffisants et des critères ont été définis pour en prioriser la distribution. Ces critères tiennent compte du risque de complications importantes pour les personnes malades avec atteinte respiratoire et la difficulté de leur prise en charge en cas de contamination par le coronavirus. La population prioritaire est donc celle des personnes trachéotomisées ou ventilées 
ayant recours à de l'aide humaine en emploi direct, par un service prestataire et de leurs aidants familiaux non équipés de masques ou risquant d'être à court de masques et les malades ayant un proche contaminé par le coronavirus (suspecté ou confirmé).

\section{Et après le 11 mai...}

Le court-moyen terme, c'est la période qui commence au 11 mai avec le début de la sortie du confinement. Pour l'AFM-Téléthon, cette étape doit être prudente et très progressive afin de n'exposer ni les malades ni ses collaborateurs, bénévoles et salariés, à une recrudescence des risques de contamination. C'est pourquoi, dans un esprit de responsabilité individuelle et collective, nous maintiendrons le télétravail pour nos collaborateurs, partout où c'est possible, sans nuire à la dynamique des équipes et à l'efficacité des actions.

Compte tenu du retour d'expérience de ces dernières semaines, il nous parait essentiel de maintenir ce lien renforcé entre l'association et les cliniciens de FILNEMUS.

Pour les malades et les familles, la question n'est pas seulement de dire qui peut sortir du confinement sans trop de risque et qui ne le peut pas. Ce serait une approche arbitraire et simpliste d'une distinction entre deux catégories de citoyens, les "confinés" et les "déconfinés". Or dans un contexte où une telle situation pourrait durer plusieurs mois voire davantage, cette vision doit être obligatoirement interrogée :

- La population des malades neuromusculaires n'est pas homogène et les facteurs de risque la concernant ne sont pas encore suffisamment définis sur des critères scientifiquement solides. Elle comporte aussi bien des personnes à très haut risque que des personnes à faible risque, selon la gravité de la pathologie neuromusculaire et son évolutivité, bien qu'ils soient globalement supérieurs à ceux de la population française. Il n'y a rien de comparable entre un malade atteint d'une myopathie de Duchenne évoluée, trachéoventilé, et par exemple certaines neuropathies périphériques qui présentent, statistiquement parlant, un risque cardio-respiratoire beaucoup plus réduit. S'agissant de maladies évolutives, comme le sont beaucoup de maladies neuromusculaires, la question de l'âge est, peut-être davantage encore que dans la population générale, un facteur dramatique d'aggravation du pronostic, les risques cardiorespiratoires s'amplifiant avec l'évolution de la maladie neuromusculaire jusqu'à parfois une issue fatale. En conséquence, la sortie de confinement d'une personne atteinte d'une maladie neuromusculaire ou de son entourage familial représente une vraie mise en danger avec un risque de contracter la COVID-19.

- Ces deux catégories ("confiné" et "non-confiné") ne peuvent être physiquement totalement séparées. Les personnes dépendantes fragiles ont besoin de contacts rapprochés avec des personnes "déconfinées" (auxiliaires de vie, autres membres de la famille, soignants...). Plus le temps passera, plus ces contacts nécessaires vont devoir se multiplier (épuisement des aidants, accès aux soins jusqu'ici repoussés, nécessité psychologique de sortir d'un espace restreint, etc.).

- Les réalités de résidence sont très différentes selon que les personnes se trouvent en établissement ou à domicile. Et parmi ces dernières certaines vivent seules, d'autres en couple ou en famille, et les conditions d'exiguiité de l'habitation et de revenus sont très diverses.

- La capacité de résistance des personnes et des familles, bien qu'élevée, a forcément des limites. La perspective d'une poursuite du confinement dans les mêmes conditions qu'actuellement sera de plus en plus difficile à supporter, y compris dans les familles où se trouvent des personnes à très haut risque.

L'approche qu'il faut retenir dans le cadre de la sortie de confinement doit plutôt être de savoir comment, dans la durée, protéger aux mieux les personnes en fonction des risques qu'elles encourent, soit directement à cause de la COVID-19, soit indirectement en raison des conséquences de renoncement aux soins et aux indispensables interactions sociales.

Cette protection, pour être acceptée et efficace, peut passer par des mesures de confinement prolongé mais ses modalités doivent pouvoir être modulées en fonction des situations.

Un travail de pédagogie, d'information, de transparence mais aussi d'anticipation est prioritaire. Afin de pouvoir prendre leurs décisions, les personnes concernées doivent avoir une visibilité sur les conditions et la durée prévisible des différentes mesures. Pour être acceptables, elles doivent être comprises comme des mesures de protection. Il faut donner les moyens aux personnes de pouvoir faire des choix éclairés pour eux et pour l'ensemble de la collectivité. Et comme il ne saurait y avoir de mesure légalement obligatoire restreignant les libertés individuelles d'une catégorie de citoyens, c'est sous la forme de recommandations et d'un appel à la responsabilité individuelle de chacun que des mesures de ce type pourront être envisagées. Pour sa part, l'AFM-Téléthon fera des recommandations aux familles neuromusculaires faisant appel à leur responsabilité à l'aune des risques qu'elles encourent. La recommandation d'un prolongement du confinement et du respect des mesures barrières, sous une forme volontaire et adaptée à chacun, nous semble à ce jour une nécessité.

En pratique, il faut dès à présent garantir un accès prioritaire et gratuit aux équipements de protection individuelle (EPI) pour toutes les personnes à risque et leurs aidants, qu'ils soient familiaux ou salariés. La nature de ces 
matériels peut être modulée en fonction des risques mais elle doit être une priorité absolue. Malgré les mesures gouvernementales à ce sujet, la situation observée actuellement n'est pas du tout satisfaisante. En particulier, les aidants salariés en emploi direct n'ont qu'un accès très partiel aux masques, avec une grande hétérogénéité de pratiques selon les régions. Par ailleurs, les aidants familiaux qui se sont substitués aux aidants professionnels par mesure de prévention pour la personne fragile n'ont, eux, aucun accès aux EPI. En termes d'accès à ces moyens de protection et tout particulièrement aux masques, les personnes fragiles, leurs aidants et leurs enfants ou leur fratrie, ne peuvent, dans la durée être considérés comme le reste de la population française et doivent être alimentés, a minima en masques chirurgicaux pour le plus grand nombre, et en masque FFP2 pour les aidants accompagnant un malade trachéoventilé ou sous ventilation non invasive.

En parallèle, il faut également garantir un accès prioritaire et gratuit aux tests PCR et immunologiques pour les personnes à risques et leurs aidants familiaux et salariés afin qu'elles puissent adapter les mesures de protection en fonction de leur statut vis-à-vis de la COVID-19.

Au niveau médical, la poursuite ou la reprise de soins déprogrammés doit être une priorité. Pour les personnes à risque, des organisations spécifiques doivent être mises en place afin que les prises en charge à l'hôpital, notamment en consultation et en hôpital de jour, puissent se faire en toute sécurité. La suppression des temps d'attente et des dérogations pour l'utilisation de transports sanitaires sont nécessaires. En particulier :

- Les suivis en centre d'expertise, centre de référence ou de compétences, doivent reprendre avec pour chaque malade l'établissement d'un bilan des conséquences de la période de confinement et des mesures de compensation à mettre en place le cas échéant.

- Les activités de diagnostic, notamment au niveau moléculaire, doivent reprendre. La lutte contre l'errance diagnostique reste un enjeu majeur dans le monde des maladies rares et il ne faudrait pas qu'elle soit sacrifiée au motif de la mobilisation des ressources pour faire face à la multiplication intensive des tests COVID-19.
- Les interventions chirurgicales qui ont été différées doivent être rapidement reprogrammées.

- Les thérapies innovantes administrées dans le cadre hospitalier, comme par exemple les injections de nusinersen pour l'amyotrophie spinale, doivent être, lorsqu'elles ont été différées, réactivées en urgence. Un bilan fonctionnel et respiratoire doit être établi à la reprise des injections. - enfin, pour certaines pathologies neuromusculaires à impact cognitif (myopathie de Duchenne, Steinert) une évaluation des conséquences de l'isolement social doit être réalisée avec à la clé d'éventuelles mesures de soutien psychologique adaptées.

La période qui s'ouvre n'est pas la fin de la crise épidémique mais une nouvelle période où nous allons vraisemblablement continuer de cohabiter avec ce virus avec le risque accru majeur qu'il représente pour la population des malades neuromusculaires. Notre challenge collectif sera à la fois de reprendre une pratique de médecine d'excellence pour ces malades, un accompagnement soutenu au quotidien, tout en maintenant un arsenal de mesures de prévention drastiques pour cette population, jusqu'à, si nécessaire, une recommandation de poursuite du confinement dans les situations les plus à risque. En veillant aussi à ce que les effets délétères d'un confinement prolongé ne soient pas supérieurs aux risques que représenterait une infection par la COVID-19. Un défi immense que l'action collective et partenariale entre tous les acteurs, scientifiques, médecins et associations de malades doit nous permettre de relever.

\section{All united against COVID-19}

Christian Cottet Directeur Général, AFM-Téléthon, Éury, France ccottet@afm-telethon.fr

\section{LIENS D'INTÉRÊT}

L'auteur déclare n'avoir aucun lien d'intérêt concernant les données publiées dans cet article.

\section{RÉFÉRENCE}

1. Solé G, Salort-Campana E, Pereon Y, Stojkovic T, Wahbi K, Cintas P, Adams D, Laforêt P, Tiffreau V, Desguerre I, Pisella LI, Molon A, Attarian S; FILNEMUS COVID-19 study group. Guidance for the care of neuromuscular patients during the covid 19 pandemic outbreak. Rev Neurol (Paris) $2020 \mathrm{Apr}$ 20. pii : S0035-3787(20)30523-3. doi : 10.1016/j.neurol.2020.04.004. 